\title{
Los menores con discapacidad como víctimas de maltrato infantil: una revisión
}

\section{Children with disabilities as child abuse victims: a review}

\section{Ana BERÁSTEGUI PEDRO-VIEJO* Blanca GÓMEZ-BENGOECHEA*}

\section{RESUMEN}

La protección adecuada de los derechos de los menores con discapacidad es todavía una asignatura pendiente de nuestras sociedades. El objetivo de esta revisión es aumentar la conciencia de los profesionales de la intervención psicosocial del mayor riesgo que sufren los menores discapacitados de sufrir cualquier tipo de abuso, no solo por factores asociados a su situación personal, familiar y social, sino también por la mayor dificultad de los profesionales para detectar las situaciones de maltrato, dar credibilidad a las sospechas sobre el mismo, denunciarlas, y tratar adecuadamente las situaciones en los que el maltrato efectivamente se produce, especialmente cuando se produce en menores con discapacidad psíquica. Se plantea que el reconocimiento de esta mayor vulnerabilidad y sus causas entre los profesionales relacionados con la infancia y/o la discapacidad es el primer paso para la prevención y la detección temprana y eficaz de estas situaciones.

\section{PALABRAS CLAVE}

Maltrato, Menores, Niños, Discapacidad, Negligencia, Factores de riesgo, Protección de menores.

\section{ABSTRACT}

The appropriate protection of children's with disabilities rights is already a non addressed issue in our societies. The main objective of this review is to increase the awareness of

\footnotetext{
* Instituto Universitario de la Familia. Universidad Pontificia Comillas de Madrid.
} 
the professionals about the greater risk of abuse and neglect in children with disabilities. This special risk is not only explained by personal, family or social factors, but also for the greater difficulties to detect the abuse situations, to evaluate the credibility of suspects, to report the suspected abuse and to give the appropriate treatment of those that the abuse indeed takes place, especially when the victims are children with learning disabilities. The recognition of this greater vulnerability and their causes among professionals related with child protection and disability will be the first step for the prevention and the early and effective detection of these situations.

\section{KEY WORDS}

Abuse, Children, Disabilities, Neglect, Risk factors, Child protection. 
La infancia con discapacidad ha sido, y sigue siendo, la población cuyos derechos han sido más impunemente negados y violados a lo largo de la historia y en la mayoria de las culturas, y, con toda probabilidad, la más vulnerable a todo tipo de maltrato. El infanticidio, el aislamiento en instituciones y el abandono familiar de los menores con discapacidad han sido ampliamente aceptados en nuestras sociedades hasta hace bien poco. Si pensamos en otros grupos que han sido vulnerables por cuestiones de raza, de sexo o de nacionalidad, los discapacitados han sido, dentro de estos colectivos, aquellos sobre los que ha recaido de manera más contundente el peso de la discriminación. El reconocimiento de los derechos de todos los niños y la preocupación por protegerlos han sido bastante tardíos históricamente, pero cuando nos referimos a los menores con alguna discapacidad, la protección adecuada de sus derechos sigue siendo una asignatura pendiente de nuestra sociedad, a pesar de los enormes avances que se han hecho en este sentido en las últimas décadas (Morris, 1998).

La relación que existe entre maltrato infantil y discapacidad se ha documentado en tres direcciones básicas en los estudios dedicados a esta cuestión: el menor discapacitado como víctima del maltrato, la discapacidad como consecuencia del maltrato infantil y, por último, el discapacitado como actor del maltrato activo y pasivo hacia otros menores (Verdugo y Bermejo, 1995).

Esta revisión teórica se centrará en el menor discapacitado como víctima de maltrato. Sin embargo, no debemos olvidar las otras dos direcciones de estudio que, a su vez, acaban incidiendo también en el aumento de la vulnerabilidad de los propios menores con discapacidad.
En este sentido, es importante resaltar cómo un importante porcentaje de discapacitados lo son como consecuencia de distintas formas de maltrato en la infancia. Sobsey (1994) sitúa este porcentaje en el 14\%, y se ha observado cómo esta relación se convierte en un círculo vicioso en el que el maltrato crea o agrava una discapacidad que, a su vez, aumenta el riesgo de maltrato (Jandes y Diamond, 1985). Es el caso, por ejemplo, del maltrato prenatal. La negligencia en el seguimiento médico del embarazo o el consumo de sustancias tóxicas durante el mismo, reconocidos por los expertos como formas de maltrato (Observatorio de Infancia, 2006), pueden derivar en síndrome alcohólico fetal o en otros daños neurológicos y funcionales que derivan en discapacidad (Zelenko, Lock, Kraemer y Steiner, 2000). También se han documentado los efectos de la negligencia afectiva y del abandono temprano en la lentificación e incluso el estancamiento del desarrollo físico y psicológico del niño (Beckett y cols., 2003; Groza y Ryan, 2002 ), y, por supuesto, del maltrato físico como generador de lesiones que pueden derivar en discapacidad (Sullivan y Knuston, 1998).

Por otro lado, se ha estudiado a los discapacitados como autores de maltrato infantil. En este sentido, se ha detectado el mayor riesgo que tienen los adultos con discapacidad intelectual de tener un comportamiento negligente 0 agresivo hacia sus hijos (McGaw y Sturmey, 1993). También se ha destacado la sobrerrepresentación de los discapacitados intelectuales como abusadores sexuales (Beail y Warden, 1995; Day, 1993; O,Callaghan, 1998), lo que a su vez se relaciona con el mayor riesgo de sus compañeros a sufrir abuso cuando viven en instituciones residenciales 0 conviven en centros escolares y dispositivos de ocio y tiempo libre. Es posible 
que la sobrerrepresentación de los discapacitados psíquicos como abusadores sexuales se deba, al menos en parte, a que este tipo de abuso se detecta de modo mucho más evidente cuando el abusador tiene discapacidad, por ser su comportamiento más impulsivo, más público y menos cuidadosamente escondido (Thompson y Brown, 1997), dándose así el efecto inverso en la probabilidad de detección que cuando el abuso se perpetra por parte de una persona sin discapacidad.

\section{EL MENOR CON DISCAPACIDAD, UNA VÍCTIMA FRECUENTE}

Tanto los enfoques teóricos como las investigaciones que se han centrado en estudiar al menor discapacitado como víctima de maltrato, tienden a encontrar que los niños con discapacidad tienen mayor vulnerabilidad frente a diversas formas de abuso (Crosse y Cols, 1993; Marchant y Page, 1992; Sullivan y Cork, 1996; Sullivan y Knuston, 1998; Verdugo, Bermejo y Fuertes, 1993, Wescott y Cross, 1996).

En España son muy pocos los estudios que han tratado de cuantificar este riesgo. En Castilla León, Verdugo y cols. (1993) encontraron que un $11,5 \%$ de los niños de una muestra de 445 discapacitados entre 0 y 19 años, habian sufrido algún tipo de maltrato a lo largo de su vida, mientras que la tasa de maltrato infantil se sitúa en España en torno al $1,5 \%$ de los menores.

También contamos con estudios de corte clínico, como el de Oliván (2002) que determina que el 5,56\% de los 1.115 menores ingresados por malos tratos intrafamiliares en centros de acogida de la provincia de Zaragoza, tenian alguna discapacidad. Teniendo en cuenta que la población discapacita- da supone un $10 \%$ de la población infantil, la subrerrepresentación entre los menores maltratados podría ser indicativa de un menor riesgo de estos menores a sufrir alguna forma de maltrato, aunque, más probablemente, estemos ante la realidad de la falta de detección, de denuncia y de intervenciones protectoras frente al maltrato cuando el menor que lo sufre tiene una discapacidad.

Si acudimos a los estudios estadounidenses, más numerosos en este ámbito, encontramos un aumento del 1,7\% en la incidencia del maltrato intrafamiliar en menores discapacitados frente al resto de los niños (Crosse y cols., 1993; Sullivan y Knutson, 1998).

La presencia de múltiples discapacidades aumenta, aún más, el riesgo de maltrato tanto activo como pasivo y además el riesgo de maltrato acompaña a esta población, no sólo a lo largo de la infancia, sino también en la vida adulta, especialmente cuando la discapacidad es psíquica (Cooke y Standen, 2002).

Si en los estudios sobre maltrato infantil se habla de que el maltrato detectado no es más que la punta del iceberg de una realidad más amplia (Morales y Costa, 1997), en el caso del maltrato a niños con discapacidad, las cifras disponibles pueden ser consideradas aún menos representativas. Las mayores dificultades de detección de este tipo de maltrato (Mitchelli, Turbille y Trunbull, 1999), y el hecho de que en las estadísticas sobre maltrato infantil no se registre adecuadamente la posible discapacidad, hace que esta realidad sea invisible para la mayor parte de la sociedad y, por tanto, su prevención menos efectiva. 


\subsection{Tipos de maltrato y contexto}

En el entorno de la protección a la infancia existe un amplio consenso en definir el maltrato como "toda acción u omisión no accidental que impide 0 pone en peligro la seguridad de los menores de 18 años y la satisfacción de sus necesidades físicas y psicológicas básicas" (Palacios, Jiménez, Oliva y Saldaña, 1998). Sin embargo, son muchas las realidades que se recogen dentro de esta definición: el abandono o negligencia, el maltrato emocional, el maltrato físico, el abuso sexual, el maltrato prenatal, la mendicidad, la corrupción, la explotación laboral, el síndrome de Münchhausen por poderes o el maltrato institucional (Palacios y cols., 1998).

Una de las preguntas pertinentes es si los menores con alguna discapacidad son vulnerables por igual a todo tipo de maltrato y en todos los contextos de desarrollo. Aunque algunos estudios encuentran que los menores con discapacidad sufren los mismos tipos de maltrato y en proporción similar al resto de la población infantil (Olivan, 2002), la mayoría de los estudios encuentran una mayor incidencia del abuso sexual, especialmente en menores con discapacidad intelectual. Por ejemplo, Sullivan y Cork (1996) detectan, en población estadounidense, un aumento del $2,2 \%$ en la incidencia de abuso sexual, un $1,8 \%$ en la incidencia del comportamiento negligente y un $1,6 \%$ en la incidencia de maltrato físico. El estudio de Verdugo y cols. (1993) encuentra que en un $2 \%$ de su muestra de discapacitados se había detectado abuso sexual y en un 4\% había sospechas de abuso, y Kennedy (1996) expone que dos de cada tres chicas y uno de cada tres chicos con discapacidad ha sufrido algún tipo de abuso sexual (Kennedy, 1996).
En cuanto al contexto, hay que mencionar que la mayor parte de los casos de malos tratos a personas con discapacidad se produce en el entorno doméstico, al igual que ocurre en la población infantil sin discapacidad (Marge, 2003; National Center on Child Abuse and Neglect, 2003; Oliván , 2002; Sobsey, 1994; Sullivan y Knutson, 2000; Ticoll, 1994; Verdugo y cols., 1993)

\subsection{Tipos de discapacidad y maltra- to}

Cuando hablamos de discapacidad, tanto en niños como en adultos, estamos refiriéndonos a una gran diversidad de situaciones. El carácter físico, psíquico o sensorial de la discapacidad sitúa a los menores en contextos evolutivos muy diferentes. Parece que el aumento de incidencia del maltrato se produce en cualquier tipo de discapacidad aunque se ha observado que el abuso es más frecuente en discapacitados psíquicos que en otros tipos de discapacidad (Cooke y Standen, 2002). Este aumento del riesgo de maltrato en discapacitados psíquicos es especialmente evidente cuando nos referimos al abuso sexual, que tiene una mayor incidencia en discapacitados psíquicos de grado medio (Hernández, Horno y Santos, 2002).

Por el contrario, parece no existir una mayor incidencia de situaciones de maltrato, ni familiar, ni escolar, ni entre iguales, en población con discapacidad visual. Incluso, se ha encontrado cómo el hecho de ser ciego total disminuía la probabilidad de sufrir maltrato entre iguales en los centros escolares de la Comunidad de Madrid (Eguren, Gutiérrez, Herrero y López, 2006). 
A pesar de que otros estudios, como el de Olivan (2002), no apoya la hipótesis de una diferente distribución del riesgo de maltrato en función del tipo de discapacidad, en este artículo pondremos especial atención en los factores de riesgo y las dificultades específicas en el trabajo con menores con discapacidad intelectual, por el mayor riesgo y también la mayor especificidad que tiene este colectivo en relación con esta cuestión.

\section{2. ¿QUÉ AUMENTA EL MALTRATO DE LOS MENORES CON DISCAPACIDAD?}

La infancia con discapacidad se ve afectada por los mismos factores de riesgo que la población infantil en general: la exclusión, el estrés de la familia, las dificultades económicas, el aislamiento social, los conflictos conyugales o la historia familiar de abuso en los padres, son factores, entre otros muchos, que aumentan el riesgo de estos menores a sufrir algún tipo de maltrato (Observatorio de Infancia, 2006). De hecho, la discapacidad no puede ser considerada por sí misma un factor desencadenante del maltrato si no se da en interacción con otras variables de riesgo como ocurre en la mayoría de estos menores cuando son maltratados (Olivan, 2002).

Junto a la incidencia de estas variables, se pueden detectar factores familiares, educativos y contextuales, así como los unidos a las necesidades especiales, que afectan específicamente a la población con discapacidad haciéndola más vulnerable al maltrato.

\subsection{Factores de vulnerabilidad del niño o la discapacidad}

Hay algunas cuestiones asociadas a la propia discapacidad que aumentan el riesgo de sufrir episodios de maltrato, dificultan el reconocimiento de los mismos por parte del menor, o dificultan que el menor pueda defenderse inmediatamente frente al maltrato o denunciarlo posteriormente.

Entre los primeros factores encontramos que la mayor necesidad de atención en el cuidado físico o la higiene personal de muchos de los menores con discapacidad les coloca en situaciones propicias para el abuso sexual (American Academy of Pediatrics, 2001; Ellis y Hendry, 1998; Hernández y cols, 2002; Morris, 1998). De hecho, el riesgo aumenta con la cantidad, y la mayor intimidad, del cuidado necesitado por el niño (Briggs, 1996). Estos menores, en muchos casos, se encuentran a cargo de personas diferentes, que cambian con cierta frecuencia, lo que, por un lado, aumenta la posibilidad de sufrir abuso por parte de alguna de estas personas, y, por otro lado, reduce el estrés que tendría un solo cuidador $\mathrm{y}$, por tanto, la probabilidad de maltrato intrafamiliar (American Academy of Pediatrics, 2001).

Además, el hecho de estar al cuidado de varias personas, especialmente si cambian con cierta frecuencia, dificulta que estos menores puedan discriminar quién es un extraño al que no se le debe permitir ciertos tipos de contacto y quién no lo es. Hernández y cols. (2002) exponen cómo en un programa de prevención del abuso sexual para discapacitados psíquicos se necesitaron 10 sesiones de trabajo para que distinguieran un extraño de alguien que no lo era. Esta dificultad para establecer distancia y límites afectivos con los extraños pone en riesgo a los menores y dificulta el reconocimiento de determinadas conductas como inadecuadas o lesivas.

Entre las dificultades para el reconocimiento de la acción abusiva como tal encontramos también, en parte por el 
mayor contacto físico de los cuidadores con el menor, una mayor dificultad para establecer la frontera entre el propio cuerpo y el cuerpo del otro y, con ello, de distinguir lo que es un contacto físico normal de un contacto abusivo, no sólo en el ámbito del abuso sexual, sino también del maltrato físico. Se ha destacado, por ejemplo, cómo el carácter doloroso de algunos tratamientos de rehabilitación o estimulación hacen que los menores tengan dificultades para distinguir el dolor adecuado del inadecuado y, por lo tanto, les cueste reconocer cuándo están siendo tratados de alguna dolencia y cuándo maltratados (American Academy of Pediatrics, 2001).

El aumento de la dificultad para reconocer el abuso y el maltrato para estos menores, no sólo dificulta la detección y la denuncia de estas situaciones, sino que puede ser, por sí mismo, una situación de riesgo. Esta dificultad puede generar en el maltratador cierta sensación de impunidad al pensar erróneamente que si la víctima no reconoce el abuso como tal, no sólo no será denunciado, sino que tampoco está generando daño y por tanto haciendo nada malo ni contrario a su dignidad (American Academy of Pediatrics, 2001).

En tercer lugar, los menores con alguna discapacidad tienen en ocasiones dificultades especiales tanto para oponerse al maltrato como para comunicarlo y denunciarlo. La mayoría de ellos tienen mayores dificultades fisicas para oponerse. Existe además, en algunos menores, una mayor dificultad emocional para poner en riesgo una relación de la que se depende vitalmente, o para oponerse a la autoridad cuando han sido educados en la sumisión, la dependencia y la obediencia, como veremos más adelante (American Accademy of Pediatrics, 2001). Cuando la discapacidad comporta graves dificultades de comunicación, existen también mayores dificultades para quejarse adecuadamente o para denunciar a pesar de que se reconozca el maltrato como tal (Morris, 1998).

\subsection{Factores familiares}

En las familias que tienen hijos con alguna discapacidad podemos encontrar un aumento de las fuentes de estrés familiar, una dificultad para acceder 0 capacitarse con recursos de afrontamiento adecuados y una mayor dificultad para evaluar la situación en términos positivos. Estas dificultades predicen un mayor riesgo de que la familia emprenda respuestas poco adaptativas como la negligencia o el maltrato, según los modelos de estrés y afrontamiento familiar (Hill, 1949; McCubbin y Paterson, 1983; McCubbin, Thompson y Former, 1998)

En este sentido tener un hijo con una discapacidad aumenta las fuentes de estrés emocionales, físicas, económicas y sociales de las familias (Benedict y cols, 1990; Hernández y cols, 2002). Las necesidades especiales del niño se suman a las necesidades de cualquier niño, disparando el nivel de demandas que tiene que afrontar la familia y el estrés (Ammerman y cols, 1993), que puede derivar en maltrato activo. Por otro lado es más fácil ser negligente con las necesidades del niño, porque son más y, en ocasiones, desconocidas para la propia familia (Hernández y cols, 2002). En definitiva, el aumento de las demandas que van unidas a la discapacidad aumenta el riesgo de maltrato, porque su desconocimiento puede conducir a la negligencia y su conocimiento a un aumento del estrés que favorece la agresión fisica (American Academy of Pediatrics, 2001; Sullivan y Cork, 1998).

La evaluación de la situación estresante en términos positivos también se ve 
dificultada en estas familias. En primer lugar, el desequilibrio entre las expectativas y la realidad se da desde el momento en que la familia conoce la discapacidad del niño. Cuanto mayor es el desequilibrio menor es la capacidad de la familia de regular cognitivamente el estrés que proviene de las demandas especiales (Hernández y cols, 2002). Principalemente cuando los niños son poco responsivos y reforzantes, como en el caso de los niños con trastornos del espectro autista, la familia no encuentra sentido fácilmente a los esfuerzos de afrontamiento (Sullivan y Cork, 199). Para las familias también es dificil en ocasiones no hacer atribuciones intencionales cuando sus hijos no responden a sus demandas, aunque esta falta de respuesta se derive de las dificultades cognitivas, de comunicación o motrices del menor.

Por último, es más difícil para estas familias encontrar los recursos adecuados para afrontar los retos que presenta la crianza de sus hijos. Son más los recursos necesarios y están menos accesibles socialmente. Adicionalmente es más dificil para ellas encontrar los apoyos sociales adecuados (Ammerman y cols, 1993), siendo frecuente un mayor aislamiento social de la familia (Hernández y cols, 2002).

\subsection{Factores educativos}

En cierto modo, el aumento del riesgo que viven los menores con discapacidad tiene que ver con el tipo de educación que han recibido tradicionalmente estos niños en campos como la educación de la autonomía, los afectos o la sexualidad.

En primer lugar y acentuando el grado de dependencia de partida, muchos de estos niños son educados, tanto en la familia como en la escuela, para obedecer al adulto y someterse a sus indicaciones.
Por ello se encuentra entre ellos una mayor dificultad para decir que no o para fiarse de sus propios criterios frente al criterio de los adultos (Sullivan y Cork, 1996; American Academy of Pediatrics, 2001; Hernández y cols., 2002).

En segundo lugar, en nuestra sociedad se ha tendido a negar o a patologizar la sexualidad del discapacitado, en especial del discapacitado intelectual (Amor, 1997), y por lo tanto, a considerar inútil hablar de sexualidad con ellos (Verdugo y cols., 1995 ). Esta carencia de educación sexual, deriva en que estos menores no son orientados para distinguir las muestras de atención o de cariño apropiadas e inapropiadas (American Academy of Pediatrics, 2001; Hernández y cols. 2002; Morris, 1998), para adecuar las manifestaciones afectivas al contexto y a la persona a la que se dirigen, para abrir vías de expresión adecuada de su propia sexualidad, ni para proteger su intimidad (Bailey, 1998). La negación de la sexualidad de las personas con discapacidad también deriva en la ausencia de un lenguaje adecuado para comunicar formas de maltrato como el abuso sexual (Hernández y cols, 2002) y en la escasez de programas de prevención del abuso en los distintos programas educativos que se dirigen a la infancia con discapacidad.

Estos factores de riesgo nos hablan de la importancia de generalizar los programas de educación sexual a los discapacitados, en especial a los discapacitados intelectuales, y el entrenamiento en habilidades sociales como el de Verdugo (1997), o el entrenamiento en cómo resistirse a la presión de otros de Bermejo (1999).

\subsection{Factores sociales}

Por último, existen una serie de factores contextuales relacionados con la 
visión social que existe sobre la discapacidad que los sitúan como víctimas fáciles de cualquier tipo de maltrato.

Aún persiste en nuestras sociedades una importante infravaloración de las personas con discapacidad, especialmente de las que tienen discapacidad intelectual (Ellis y Richard, 1998; Morris, 1998). En parte, esta visión negativa y marginadora sobre la persona con discapacidad es un mensaje hacia los potenciales agresores de la impunidad que tendrá el maltrato, porque a los niños con discapacidad se les reconocen, en la práctica, menos derechos que al resto (Kennedy, 1996; Morris, 1998).

Esto hace que los menores con discapacidad sean percibidos por los potenciales agresores, independientemente de si son miembros o no de su familia, como más vulnerables, menos poderosos, menos capaces de revelar el abuso y menos creíbles en el caso de hacerlo, lo que les convierte en "blancos fáciles" (American Academy of Pediatrics, 2001, Ellis y Hendry, 1998). Esta concepción no solo forma parte del imaginario del maltratador sino que, efectivamente, se ha comprobado la escasa credibilidad que se otorga a sus testimonios y denuncias (Sobsey y Vamhagen, 1989). Como en todos los casos de maltrato infantil, pero quizás aún más agudizado, la falta de confianza en el testimonio de estos niños está basada en un deseo de no pensar lo impensable (Brown y Craft, 1989).

Aun cuando los menores son atendidos en sus sintomas y se presta credibilidad a su testimonio, muchas veces no se interviene por la falsa creencia de que el maltrato afecta menos a un menor con discapacidad intelectual porque no se entera (Briggs y Potter, 2004; Sobsey, Randall y Parrilla, 1997; Bailey, 1998). Por ejemplo, con cierta frecuencia se toman medidas contra las consecuencias fisicas de una relación sexual no consentida esterilizando a las chicas, pero no se previene educativa ni institucionalmente frente al posible abuso.

Sin embargo, es innegable que el abuso produce consecuencias negativas en el discapacitado intelectual como el aumento en la sensación de aislamiento, la pérdida de autoestima, la ansiedad, el miedo, y los sentimientos de incapacidad, vergüenza, depresión, culpa, frustración, miedo y estigmatización (Briggs, 1996; Bayley, 1998; Ammerman y cols, 1994). Incluso algunos niños llegan a estados psicóticos teñidos por la confusión y la rabia (Verdugo y cols., 1993).

Por último hay un factor social asociado al tipo de dispositivos que se generan para la atención de los menores con discapacidad. Aunque la institucionalización es un recurso cada vez menos frecuente en la mayoría de las discapacidades, aún podemos encontrar unidades residenciales (aunque son más frecuentes en adultos que en niños) que dejan a estas personas en una situación de cierto aislamiento, lejos de la comunidad y de las fuentes de apoyo (Ellis y Hendry, 1998). Por ejemplo, un cuarto de los discapacitados víctimas de abusos sexuales son atacados por usuarios masculinos de sus mismos centros residenciales.

\section{OBSTÁCULOS PARA LA PROTECCIÓN ADECUADA DE LOS MENORES DISCAPACITADOS FRENTE AL MALTRATO}

A pesar de el enorme trabajo que se ha realizado en las últimas décadas con respecto a la protección de los menores frente al maltrato, aún encontramos ciertas barreras a la detección y denuncia de estas situaciones, independientemente de que los niños tengan o no discapacidad. Algunas de estas barreras 
son la falta de formación de los profesionales implicados en el trabajo con menores, la falta de confianza en que los indicios de maltrato sean suficientes para poner una denuncia, el miedo de los posibles denunciantes a que se produzcan represalias contra el niño o contra ellos mismos, la dificultad de los profesionales para poner en riesgo una relación de ayuda que se haya podido establecer con la familia maltratadora si se denuncia el maltrato, y la falta de confianza en la acción de los servicios de protección frente a una eventual denuncia. En el fondo, el miedo a que para el niño sea peor el remedio que la enfermedad (Micheli y cols, 1999).

Todos estos obstáculos se dan de un modo quizás todavía más acusado cuando se trata de menores con discapacidad. En estos casos se ha encontrado que no se detecta adecuadamente, no se denuncia todo lo que se detecta y no se trata adecuadamente todo lo que se denuncia (Cooke y Standen, 2002).

\subsection{No se detecta adecuadamente}

Ya hemos mencionado algunas de las dificultades adicionales en la detección de situaciones de maltrato a niños con discapacidad: los niños pueden estar limitados a la hora de reconocer y comunicar el abuso y se presta menos credibilidad a sus testimonios cuando lo cuentan.

Adicionalmente, los profesionales se encuentran con ciertas dificultades para distinguir los signos y sintomas del abuso de los que están causados por la propia discapacidad ( American Academy of Pediatrics, 2001, Cooke y Standen, 2002; Garbarino y Authier, 1987; Micheli y cols., 1999). Por ejemplo se considera que el comportamiento sexualizado precozmente, que puede ser considerado un signo de abuso sexual, puede ser conse- cuencia de la propia discapacidad (Cooke y Standen, 2002), o que los golpes y moratones de los niños pueden estar derivados de su inestabilidad motora, o incluso, en algunos tipos de discapacidad, de autolesiones esperables.

Por otro lado, no sólo se presta menos credibilidad a los menores con discapacidad y a sus sintomas que a los que no la tienen, sino que se presta una mayor credibilidad y se suele conceder el beneficio de la duda a los padres de estos niños, por la empatía que genera la situación que tienen que afrontar (Cooke y Standen, 2002). En este sentido, a los profesionales les resulta más difícil considerar intencional el daño que unos padres pueden infligir a su hijo con discapacidad, especialmente cuando se trata de negligencia pero también en casos de maltrato físico (Michelil y cols, 1999). También resulta dificil para algunos profesionales distinguir entre abuso y disciplina, que aumenta cuanto mayores son las dificultades de comunicación y de lenguaje del niño y, por tanto, más conductuales deben ser las intervenciones educativas (Micheli y cols., 1999).

$\mathrm{Si}$, por lo general, los criterios de abuso son vagos y las definiciones de negligencia aún más, encontramos que los profesionales a cargo de los menores no tienen la formación específica para aplicar estos parámetros cuando nos referimos a niños y niñas con necesidades especiales (Micheli y cols, 1999). Todo esto hace que el abuso no sea reconocido hasta que los sintomas son muy graves y el daño muy importante para el niño (Cooke y Standen, 2002).

\subsection{No se denuncia lo que se detecta}

En el campo de la discapacidad se exacerba el miedo de los profesionales a 
que denunciar sea aún peor para el niño que la situación de maltrato (Micheli y cols, 1999). A esto se añade la sensación que ya hemos mencionado, especialmente en casos de abuso sexual a discapacitados intelectuales, de que las consecuencias del maltrato son menos graves en los niños con discapacidad.

De nuevo, la relación empática y de comprensión que los profesionales suelen establecer con estas familias, agrava la dificultad para denunciar, por las barreras que se anteponen a romper la relación con una familia por la que se siente empatía y con la que se ha establecido una relación de ayuda.

\subsection{No se trata adecuadamente lo que se denuncia}

Parece que la falsa creencia de que a los menores con discapacidad les afecta menos el maltrato puede estar presente también en el tipo de tratamiento que se da una vez se pone en conocimiento de los servicios de protección las situaciones de maltrato. Cooke y Standen (2002) encuentran, por ejemplo, que los menores con discapacidad que sufren maltrato son más frecuentemente derivados a tratamiento e investigación médica pero, sin embargo, se abren menos expedientes de protección, menos investigaciones penales, se programan menos terapias, menos cambios de residencias y menos programas de apoyo familiar cuando el menor tiene discapacidad. De este modo parece que se atienden las necesidades físicas de los menores pero se obvian las emocionales, quizás porque no se saben abordar adecuadamente 0 porque no existen dispositivos especializados para ello.

En cuanto a la protección legal que reciben los menores discapacitados víctimas de maltrato no es diferente de la que asiste al resto de menores. Nuestro ordenamiento jurídico prevé algunas especialidades en la legislación administrativa de protección, la penal y la procesal, para las situaciones en las que están implicadas personas incapacitadas (casi siempre equivalentes a las establecidas para menores). Sin embargo, una y otra no se acumulan en los casos en los que ambas condiciones se dan juntas, de manera que la protección legal que recibe frente al maltrato un menor discapacitado es la misma que recibiría un menor sin ninguna discapacidad.

En relación con la protección jurídica de la que son objeto estos menores es importante distinguir entre las actuaciones tipificadas como delito en el Código Penal, y las cuestiones procesales, que tienen que ver con la participación de los menores víctimas de maltrato en los procedimientos judiciales en los que se juzga a sus presuntos agresores.

Con respecto a la cuestión penal, no existe un delito específico en el que se tipifique el maltrato a menores y/o personas discapacitadas, sino que se prevén penas especiales para los casos en que determinados delitos tienen como víctimas a menores o personas que se encuentran en especial situación de vulnerabilidad. Así ocurre en los delitos de abandono, lesiones, maltrato familiar, y los delitos contra la libertad o indemnidad sexual.

Con respecto al tratamiento que reciben los menores discapacitados durante el procedimiento judicial que sigue a la denuncia (en los casos en los que ésta se produce), se ha denunciado reiteradamente, con frecuencia en relación con los casos de abuso sexual, que los niños son objeto también de un importante maltrato institucional y victimización secundaria.

Los procedimientos penales en los que se enjuician estas conductas tienen 
como objetivo castigar al autor, incluso por encima de la protección y de poner remedio a la situación del menor, de manera que el procedimiento termina volviéndose contra la víctima, generándole un importante estrés y ansiedad cuando tiene que actuar como testigo. (Del Molino, Horno y Santos, 2002).

La necesidad de declarar sobre la misma cuestión en varias ocasiones y a lo largo de varios meses, que obliga a mantener abierta la herida, y la duda tanto sobre la credibilidad del testimonio de los niños, que muchas veces es la prueba fundamental y hace que sean sometidos a múltiples interrogatorios, como sobre la validez de los métodos forenses al uso para determinarla (Cederborg y Lamb, 2006), son mencionados como graves inconvenientes.

Además, en estas situaciones, los niños desconocen el procedimiento judicial, pueden encontrarse con su agresor en los Juzgados (hasta hace poco la declaración podía, incluso, realizarse cara a cara con el agresor), no entienden el vocabulario que se utiliza, y pueden, fácilmente, verse intimidados por la "escenografia" en la que se desarrolla un juicio de estas caracteristicas.

Estos inconvenientes que rodean a la declaración como testigos de los menores discapacitados víctimas de maltrato, se han visto notablemente mejorados a partir de las reformas en los procedimientos penales realizadas en 1999 y en $2006^{1}$.

Estas reformas determinan, entre otras cuestiones, que las declaraciones de los testigos menores de edad se lleven a cabo evitando la confrontación visual con el inculpado, utilizando para ello cualquier medio técnico que lo permita, y que su comparecencia en el procedimiento como testigos, inculpados o en otra condición que les resulte gravosa 0 perjudicial, se realice a través de videoconferencia u otro sistema similar (Arts. 707, 731 bis, 448 LECr) ${ }^{2}$.

Además, desde diciembre de 2006, la declaración de un menor podrá realizarse ante expertos, siempre en presencia del Ministerio Fiscal, y con sus padres o tutores presentes siempre que no sean imputados o el Juez acuerde lo contrario de forma motivada. Se contempla también la posibilidad de que el Juez acuerde la grabación de la declaración (Art. 433 LECr).

Desde 1999 están también eliminados los careos con testigos menores de edad salvo que el Juez o Tribunal lo considere imprescindible y no lesivo para el interés de los testigos previo informe pericial (Art. 713 LECr).

\section{5.- CONCLUSIONES}

A pesar de que se reconoce que los niños con discapacidad son especialmente vulnerables al abuso y al maltrato, aún no se han generalizado en España los programas de prevención ni de tratamiento del maltrato en esta población que deberian ponerse en marcha para mitigar la influencia de todos los factores de riesgo específicos de estos niños.

Es difícil que la administración y la sociedad estén preparadas para cubrir las necesidades de los niños con discapacidad frente al abuso y al maltrato cuan-

\footnotetext{
${ }^{1}$ L.O. 8/2006 de 4 de diciembre. L.O. 14/99, de 14 de julio.

${ }^{2}$ Desde 1999 existia la posibilidad de que el Juez, en resolución motivada y teniendo en cuenta el tipo de delito y las circunstancias, autorizara que la declaración se realizara de esta forma. Desde 2006 esta manera de declarar aparece como regla general en los casos en los que tengan que intervenir menores, y no exige resolución motivada.
} 
do no hay sensación de riesgo en la sociedad. La falta de una información fiable del número de discapacitados que han sufrido abuso, el grado y el tipo de minusvalia que tienen y el tipo de abuso que sufren es una carencia muy importante. Por ello, estas cuestiones deberian incluirse tanto en los protocolos para la Detección, Notificación y Registro de casos de Maltrato Infantil (Observatorio de Infancia, 2006) como en el Sistema de Información de Usuarios de Servicios Sociales (SIUSS). Una investigación eficaz en torno a esta realidad podrá sentar las bases de una formación adecuada de los profesionales que trabajan en el campo de la infancia y de la discapacidad para la prevención, el reconocimiento, el registro y la respuesta adecuada al abuso de los menores con discapacidad.

\section{BIBLIOGRAFÍA}

American Academy of Pediatrics (2001). Assessment of Maltreatment of Children with Disabilities. Pediatrics 108 (2), 508-512.

Ammerman, R.T., Hersen, M., Van Hasselt, V.B., Lubetsky, M.J. y Sieck, W.R. (1994). Maltreatment in Psychiatrically Hospitalized Children and Adolescents with Developmental Disabilities: Prevalence and Correlates. Journal of the American Academic Child Adolescence Psychiatry, 33 (4), 567-576

Amor, J.R. (1997). Afectividad y Sexualidad de la persona con deficiencia mental. Madrid: Universidad Pontificia Comillas.

Bailey, G. (1998). Action against abuse. Recognising and preventing abuse of people with learning disabilities. Chesterfield. UK: Association for residential care.

Beckett, C., Castle, J., Groothues, C., O-Connor, T.G., Ruter, M. y ERA Study Team (2003) Health problems in children adopted from Romania: Association with Duration of deprivation and behavioural problems. Adoption and fostering, 27 (4), 19-29.

Bermejo, B.G. (1999). El desarrollo de habilidades sociales como forma de prevenir el abuso sexual en personas con discapacidad. Siglo Cero, 30 (6), 27-31

Briggs, F. (1996). Developing personal safety skills in children with disabilities. Londres: Jessica Kingsley.

Briggs, F. y Potter, G.K. (2004). Singaporean early childhood teachers responses to myths about child abuse. Early Child Development and Care, 174 (4), 339-355.

Brown, H. y Craft, A. (1989). Thinking the Unthinkable: papers of sexual abuse and people with learning difficulties. Londres: Family Planning Association.

Cederborg, A.C. y Lamb, M.E. (2006). How does the legal system respond when children with learning difficulties are victimized?. Child Abuse and Neglect, 30 (5), 537-547.

Centro Reina Sofia para el Estudio de la Violencia (2002). Maltrato infantil en la familia, España (1997-1998). Valencia: Centro Reina Sofia.

Costa, M. y Morales, J. (1997). La prevención del maltrato infantil. En J. Casado, J.A. Díaz Huertas y M.C. Martínez (dir). Niños Maltratados (pp. 325-336). Madrid: Díaz de Santos.

Day, K. (1993). Crime and Mental Retardation. En K. Howells y H. Hollin (Eds.) Clinical Approaches to the Mentally disordered ofender. Chichester: Willey.

Eguren, P. Gutiérrez, H. Herrero, M.I. y López, J.L. (2006). Maltrato y discapacidad visual. Psicología Educativa 12 (1).

Ellis, R. y Hendry, E.B. (1998). Do we all know the score?. Child Abuse Review, 7, 360-363.

Groza, V. y Ryan, S. (2002). Preadoption stress and its association with child behaviour in domestic special needs and international adoptions. Psychoneuroendocrinology, 27, 181-197.

Hernández, M.C.; Horno, P. y Santos, A. (2002). Niños y niñas con discapacidad víctimas de abuso sexual y el procedimiento judicial. En. Sánchez, J., del Molino, C., Horno, P. y Santos, A. Niños y niñas victimas de abuso sexual y el procedimiento judicial. (pp. 82-88). Madrid: Save the Children. Accessible en www.savethechildren.es

Hill, R. (1949). Families Under Stress. New York: Harper and Row. 
Horno, P.: Santos, A.; Del Molino, C. (2002). Abuso sexual infantil. Manual de formación para profesionales. Madrid: Save the Children.

Kennedy, M. (1996). Agresiones sexuales y discapacidad infantil. En J. Morris (Ed.) Encuentros con desconocidas. Feminismo y discapacidad (pp. 139159). Madrid: Narcea.

Marge DK.(2003). A call to action: Ending crimes of violence against children and adults with disabilities. Syracuse: SUNY Upstate Medical University.

National Center on Child Abuse and Neglect. (2001). In focus: The risk and prevention of maltreatment of children with disabilities. Washington: National Clearinghouse on Child Abuse and Neglect Information, Administration for Children and Families, U.S. Department of Health and Human Services.

Mc Gaw, S. y Sturmey, P. (1993). Identifying the needs of parents with learning disabilities: a review. Child Abuse Review, 2, 101-107.

McCubbin, H.I. y Patterson, J.M. (1983). The family stress process: the double ABCX model of adjustment and adaptation. Marriage and Family Review, 6, 7-37.

McCubbin, H.I., Thompson, E.A. y Former, J.E. (1998). Stress, Coping and Health in families: sense of coherence and resilency. Thousand Oaks, CA: Sage Publications

Mitchell, L.M.; Turbiville, V. y Turnbull, H.R. (1999). Reporting abuse and neglect of children with disabilities: early childhood service providers' views. Infants and Young Children, 11 (3), 19-26.

O`Callaghan, D. (1998). Practice Issues in working with young abusers who have learning disabilities. Child Abuse Review, 7, 435-448.

Olivan, G. (2002) Maltrato en niños con discapacidades: características y factores de riesgo. Anales Españoles de Pediatria, 56 (3), 219-223

Olivan, G. (2005). ¿Qué se puede hacer para prevenir la violencia y el maltrato en niños con discapacidad?. Anales Españoles de Pediatría, 62 (2), 153-157.

Observatorio de Infancia (2006). Maltrato infantil: detección, notificación y registro de casos. Madrid: Ministerio de Trabajo y Asuntos Sociales. Accesible en: http://www.mtas.es/inicioas/observatoriodeinfancia/documentos/HojasDeteccion.pdf.
Palacios, J., Jiménez, J., Moreno, M.C., Oliva, A. y Saldaña, D.(1995). Frecuencia de los malos tratos en la infancia. En: Gómez de Terreros I, ed. Los profesionales de la salud ante el maltrato infantil.(pp. 95-119) Granada: Comares.

Palacios, J.; Jiménez, J., Oliva, A. y Saldaña, D. (1998). Malos tratos a los niños en la familia. En. M.J. Rodrigo y J. Palacios (Eds.). Familia y Desarrollo Humano (pp.399-421). Madrid: Alianza Editorial.

Sobsey, D. (1994). Violence and abuse in the lives of people with disabilities: The end of silent acceptance? Baltimore: Paul H Brookes, 1994.

Sobsey, D. y Vamhagen, C. (1988). Sexual abuse and exploitation of people with disabilities: toward prevention and treatment. En. M.C. Sapo y L. Gougen (Eds.). Special education across Canada. Centre for Human Development and research.

Sobsey, D., Randall, W. y Parrilla, R.K. (1997) Teaching people with disabilities to be abused and exploited: the special educator as accomplice. Developmental disabilities bulletin, 25 (1), 77-93.

Sullivan, P.M. y Cork, P.M. (1998). Maltreatment prevention programs for children with disabilities: An evaluation model. Developmental Disabilities Bulletin, 26, 59-71.

Sullivan, P.M. y Knutson J.F. (2000). Maltreatment and disabilities: A population-based epidemiological study. Child Abuse and Neglect, 24, 12571273.

Ticoll M. (1994) Violence and people with disabilities: A review of the literature. Ottawa: National Clearinghouse on Family Violence, Family Violence Prevention Division, Health Canada.

Verdugo, M.A. (1997). P.H.S. Programa de Habilidades sociales. Programas conductuales alternativos. Salamanca: Amarú ediciones.

Verdugo, M.A. y Bermejo, B.G. (1995). El maltrato en personas con retraso mental. En M.A. Verdugo (Dir.), Personas con discapacidad. Perspectivas psicopedagógicas y rehabilitadoras (pp. 873-924). Madrid: Siglo XXI

Verdugo, M.A., Bermejo, B.G. y Fuertes, J. y Elices, J.A. (1993). Maltrato infantil y minusvalia. Madrid. Ministerio de Trabajo y Asuntos Sociales.

Zelenko, M., Lock, J., Kraemer, H.C. y Steiner, H. (2000). Perinatal complications and child abuse in a poverty sample. Child Abuse and Neglect, 24 (7): 939-950. 